\title{
O QUE É ESSENCIAL PARA OS PROFISSIONAIS ESSENCIAIS?
}

Isabel Cristina Kowal OIm Cunha

Neyson Pinheiro Freire ${ }^{1}$ https://orcid.org/0000-0001-6374-5665.

https://orcid.org/0000-0002-9038-9974.

Objetivo: Refletir acerca do que os profissionais de Enfermagem consideram importante em seu trabalho. Método: Trata-se de um artigo de reflexão a partir de resultados de pesquisa telefônica feita pelo Conselho Federal de Enfermagem em 2020 , no início da pandemia de COVID-19. O relatório foi lido e dele coletados dados que subsidiassem o estudo. Resultados: Foram ouvidos 2.004 profissionais de Enfermagem de todo o país que apontaram como preocupações: o piso salarial, estímulo para o trabalho, cursos de qualificação e reconhecimento e respeito, que direcionaram esta reflexão. Conclusão: A despeito da visibilidade conquistada por sua atuação na luta contra o coronavírus, os profissionais de Enfermagem necessitam de maior reconhecimento do poder público. Questões há muito reivindicadas como salário adequado, insalubridade, jornada de trabalho de 30 horas, bem como melhores condições de trabalho, estão entre propostas para garantir o que é essencial para os trabalhadores essenciais, durante e depois desta pandemia.

Descritores: Enfermagem; Pandemia; COVID-19; Pessoal de saúde.

\section{WHAT IS ESSENTIAL FOR ESSENTIAL PROFESSIONALS?}

Objective: To reflect on what nursing professionals consider important in their work. Method: This is a reflection article based on the results of a phone survey carried out by the Federal Nursing Council in 2020, at the beginning of the COVID-19 pandemic. The report was read and data was collected to support the study. Results: 2004 nursing professionals from all over the country were heard, who raised concerns about basic salary, stimulus for work and qualification courses, recognition and respect, guiding this reflection. Conclusion: Despite the visibility gained by their performance in the fight against the coronavirus, nursing professionals need greater recognition from the public authorities. Issues that have long been claimed such as adequate wages, unsanitary conditions, a 30-hour working week, as well as better working conditions, are among proposals to guarantee what is essential for essential workers, during and after this pandemic.

Descriptors: Nursing; Pandemic; COVID-19; Health Personnel.

\section{¿QUÉ ES ESENCIAL PARA LOS PROFESIONALES ESENCIALES?}

Objetivo: reflexionar sobre lo que los profesionales de enfermería consideran importante en su trabajo. Método: Este es un artículo de reflexión basado en los resultados de una encuesta telefónica realizada por el Consejo Federal de Enfermería en 2020 al comienzo de la pandemia de COVID-19. Se leyó el informe y se recopilaron datos para respaldar el estudio. Resultados: 2004 profesionales de enfermería fueron escuchados de todo el país, quienes expresaron su preocupación sobre el piso salarial, estímulo para el trabajo y cursos de calificación y reconocimiento y respeto, que guiaron esta reflexión. Conclusión: a pesar de la visibilidad obtenida por su desempeño en la lucha contra el coronavirus, los profesionales de enfermería necesitan un mayor reconocimiento por parte de las autoridades públicas. Cuestiones que se han reclamado durante mucho tiempo, como salarios adecuados, trabajo no saludable, una jornada laboral de 30 horas, así como mejores condiciones de trabajo, se encuentran entre las propuestas para garantizar lo que es esencial para los trabajadores esenciales, durante y después de esta pandemia.

Descriptores: Enfermería; Pandemia; COVID-19; Personal de Salud.

IUniversidade Federal de São Paulo (UNIFESP). Grupo de Estudos e Pesquisa em Administração em Saúde e Gerenciamento de Enfermagem (GEPAG). São Paulo, SP, Brasil.

Autor correspondente: Neyson Pinheiro Freire. E-mail: neysonfreire@gmail.com.

Recebido: 08/7/20 Aceito: 18/7/20 


\section{INTRODUÇÃO}

A Emergência de Saúde Pública Global associada ao novo coronavirus, declarada pela Organização Mundial de Saúde (OMS) em 30 de janeiro de 2020 e elevada a pandemia em 16 de março, fez com que os profissionais da saúde assumissem o protagonismo das ações de cuidado aos doentes e de orientações para prevenção tanto das equipes, como de toda a população ${ }^{1-2}$. A falta de evidências científicas disponíveis sobre a nova pandemia trouxe muita inquietação. Dúvidas cruciais não podiam ser respondidas e tudo baseava-se nos resultados da experiência da China, onde a pandemia começou, até que instituições como a OMS, Ministério e Secretarias da Saúde do país começaram a emitir as primeiras orientações. Certamente esta falta de conhecimento e de informações fez com que muitas ações, desencadeadas pelas equipes de saúde, por muitas vezes fosse reticente, trazendo riscos e até contaminação a esses profissionais e à população em geral.

Os profissionais de Enfermagem respondem pela maior parte da força de trabalho em saúde no país e em todo o mundo. No Brasil, entre enfermeiros, técnicos e auxiliares de enfermagem têm-se um exército de 2,3 milhões de trabalhadores $^{3}$. Cerca da metade estão atuando na linha de frente no combate ao novo coronavírus. É o maior grupo de profissionais que lida com a doença, quando comparado com a atuação de outras categorias profissionais da área da saúde. Por conta disso, a pandemia deu visibilidade a todos eles, quer pelo cuidado direto à beira do leito aos doentes pela $\mathrm{CO}$ VID-19, quer pelas dificuldades enfrentadas no cotidiano de trabalho, como a falta ou dificuldade de acesso aos Equipamentos de Proteção Individual (EPI) de qualidade.

$\mathrm{Na}$ linha de frente do combate à doença, ao lado dos médicos e outros profissionais da saúde, os trabalhadores da Enfermagem são os mais expostos ao contágio. Convivem com uma cruel realidade da precariedade dos serviços de saúde, que os coloca em situação de extrema vulnerabilidade diante do risco de infecção. O registro de casos da doença e de óbitos não deixa dúvidas. Dados do Observatório da Enfermagem do COFEN ${ }^{4}$, atualizados em 6 de julho de 2020, mostram que mais de 23 mil casos de suspeita e confirmação e 240 mortes de profissionais de Enfermagem no Brasil com diagnóstico confirmado por COVID-19 foram reportados.

É uma situação grave, que foge ao esperado e demonstra as consequências da insuficiência e inadequação dos EPI, a relutância em afastar profissionais integrantes de grupos de risco da linha de frente do combate à COVID-19, do próprio sub dimensionamento das equipes e a sobrecarga de trabatho, que contribui para uma maior exposição.

Esta situação trouxe uma maior visibilidade política e so- cial para questões básicas de condições de trabalhos para a Enfermagem, já reivindicadas há muito tempo, tais como remuneração adequada com estabelecimento de piso salarial, jornada de 30 horas, locais de descanso no plantão para descompressão e lanche, materiais de proteção individual adequados, dimensionamento de pessoal adequado evitando a sobrecarga, entre muitos outros.

Reconhecidos como essenciais no combate à pandemia, os profissionais de Enfermagem foram trazidos ao centro do debate público no Brasil. Neste contexto, questionamos: 0 que é essencial para estes profissionais que são essenciais no cuidado, sobretudo nesta pandemia? Conhecer quais as questões de trabalho são relevantes e torna-se importante, a fim de que os gestores e as instituições possam responder de forma positiva à estas necessidades. Assim, este artigo teve como objetivo refletir acerca das necessidades dos profissionais de Enfermagem no contexto da pandemia de COVID-19.

\section{MÉTODO}

Trata-se de um artigo de reflexão a partir dos registros de levantamentos de dados do Conselho Federal de Enfermagem (COFEN), no início da pandemia, feitos por telefone com 2.004 profissionais de Enfermagem de todo o país. Foi perguntado a esses profissionais os questionamentos: "Pensando, agora, na sua atuação profissional, qual é a coisa mais importante para o profissional de enfermagem? E a segunda? E a terceira?"5

Os dados foram tabulados em relatório e utilizados para tomada de decisões pelo Plenário do Cofen e, em seguida, arquivado. Foi solicitada autorização para leitura deste material a fim de conhecer seus resultados e possibilitar esta reflexão. O relatório foi lido e foram coletados dados considerados mais relevantes.

Por ser um artigo de reflexão utilizando dados secundários sem identificação dos respondentes, não se aplica apreciação junto ao Comitê de Ética em Pesquisa, apesar de terem sido respeitos os aspectos éticos e legais neste estudo.

\section{RESULTADOS}

As respostas visualizadas na Figura 1 revelaram as preocupações essenciais dos trabalhadores da Enfermagem. $O$ piso salarial foi o tema mais citado, tendo sido apontado por $26,0 \%$ dos participantes, entre aquilo que consideravam mais importante. Tiveram destaque, também, os cursos de qualificação profissional (15,0\%), reconhecimento e respeito (14,0\%) e regulamentação da jornada de trabalho em 30 horas semanais (12,0\%). "Empatia e respeito pelos pacientes", "amor à profissão" e "melhores condições de trabalho" aparecem na sequência. 
Figura 1 - Resultados da pesquisa telefônica do COFEN, 2020.

\section{Coisas mais importantes para o profissional de enfermagem (na amostra total)}

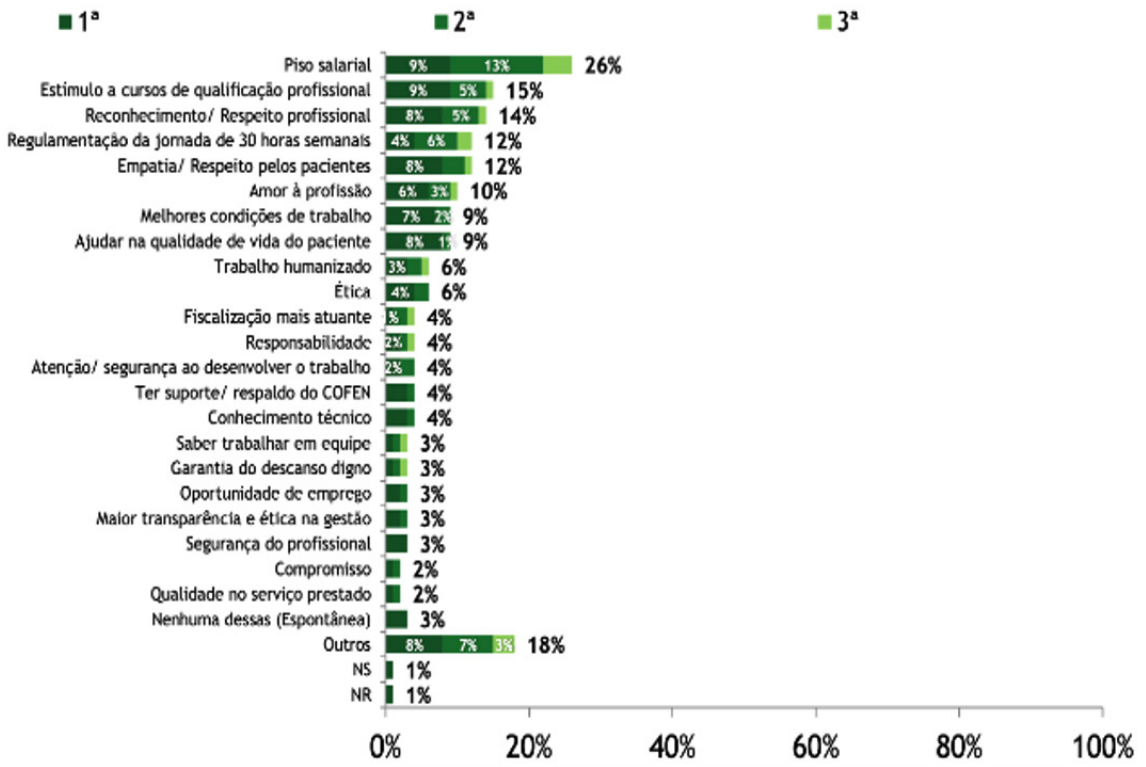

\section{Fonte: Pesquisa COFEN ${ }^{5}$.}

Dentre os temas agrupados em "outras questões", foram citados por $18 \%$ dos profissionais e estão listados na Figura 2. Destaca-se a sensibilização sobre as dificuldades vividas pelos profissionais, tais como: "qualidade de vida", "bom ambiente de trabalho", "saúde psicológica do profissional", e, ainda, outras preocupações com questões sociais e econômicas.
A centralidade dada ao tema salarial, mesmo em um contexto pandêmico, reflete uma percepção generalizada de que a importância da Enfermagem não se reflete em políticas efetivas de valorização. A prevalência de baixos salários é uma característica marcante do campo de trabalho da Enfermagem no Brasil, havendo, inclusive, indícios de saturação, como desemprego, associados a uma formação desordenada ${ }^{6}$.

\begin{tabular}{|c|c|c|c|c|c|}
\hline \multicolumn{6}{|c|}{ Outros } \\
\hline $\begin{array}{c}\text { Dimensionamento do quadro de } \\
\text { profissionais }\end{array}$ & $2,2 \%$ & Incentivo à educação continuada & $0,5 \%$ & Estabilidade & $0,2 \%$ \\
\hline Dedicação & $2,0 \%$ & Administrar medicação & $0,5 \%$ & Plano de saúde & $0,2 \%$ \\
\hline Saúde psicológica do profissional & $1,5 \%$ & Redução da anuidade & $0,4 \%$ & Humildade & $0,1 \%$ \\
\hline Bom ambiente de trabalho & $1,2 \%$ & Campanha para realização de concursos públicos & $0,4 \%$ & $\begin{array}{l}\text { Exames periódicos com } \\
\text { profissionais }\end{array}$ & $0,1 \%$ \\
\hline Autonomia & $1,2 \%$ & Auxiliar o acesso do profissional ao mercado de trabalho & $0,4 \%$ & Honestidade & $0,1 \%$ \\
\hline Aposentadoria especial & $1,1 \%$ & Maior atuação no interior do Estado & $0,4 \%$ & Organização & $0,1 \%$ \\
\hline Boa formação profissional & $1,1 \%$ & Credibilidade & $0,3 \%$ & Regulação da profissão & $0,1 \%$ \\
\hline $\begin{array}{l}\text { Melhoria dos seus canais de } \\
\text { comunicação }\end{array}$ & $1,0 \%$ & União das classes & $0,3 \%$ & Mais benefícios & $0,1 \%$ \\
\hline Competência & $1,0 \%$ & Saúde do profissional & $0,3 \%$ & Combate ao EAD na educação & $0,1 \%$ \\
\hline Profissionalismo & $0,9 \%$ & Dignidade & $0,3 \%$ & & \\
\hline Qualidade de vida do profissional & $0,7 \%$ & Contribuir com novos estudos na medicina & $0,2 \%$ & & \\
\hline
\end{tabular}

Fonte: Pesquisa COFEN ${ }^{3}$. 


\section{DISCUSSÃO}

As questões trabalhistas da Enfermagem brasileira estão entre as de maior essencialidade nesse periodo de COVID-19, com destaque para salário digno, chance de crescimento profissional, reconhecimento e respeito.

Nesse triste cenário, as condições de trabalho e de vida enfrentadas pela Enfermagem saltam aos olhos pelo negligenciar visivel da gestão pública da saúde em todas as esferas. A carência de EPI, a escassez de testes de sondagem diagnóstica e a invisibilidade histórica por parte dos governantes, descortina uma situação assustadora, que é a precarização das condições de trabalho e salário. Somado a isso, a carga emocional é ainda mais pesada para aqueles que estão vivendo longe de suas casas, de cônjuges e filhos, para poupá-los do contágio. Os cônjuges, por sua vez, vivem em um estado de medo, pois conhecem a escassez de EPI, além de lidar com uma sobrecarga de responsabilidades em casa ${ }^{7}$, no cotidiano de estresse, ansiedade, depressão e medo de adoecer e morrer.

A Organização Panamericana da Saúde (OPAS) disponibilizou um checklist de recomendações sobre a gestão dos trabalhadores da saúde durante o período de emergência global de saúde pública, com destaque para o número suficiente de profissionais e de $\mathrm{EPI}^{8}$. Todavia, estas recomendações não foram implantadas pela maioria dos serviços de saúde no Brasil, o que talvez ajude a explicar os altos índices de morbidade e mortalidade entre os profissionais de Enfermagem no país.

Essa problemática mundial da COVID-19 remete ao questionamento: Quanto vale o trabalho de quem arrisca a vida para salvar outras vidas? A Organização Internacional do Trabalho (OIT) em recente relatório sobre a pandemia aponta que no Brasil, mais de 60\% dos profissionais de Enfermagem ganham menos de dois mil reais por mês (62,2\%), e mais de um terço deles (38,7\%) têm jornadas superiores há 41 horas semanais ${ }^{9}$, configurando situações inversamente proporcionais e de precarização do trabalho.

Nesta pandemia, muitas vezes, o profissional, além de lidar com a falta de equipamentos, terem que cumprir escalas que os sobrecarregam por conta do contingente limitado de profissionais, vivenciam tomadas de decisões dificeis frente a situações como a falta de respiradores para todos os pacientes, encarando dilemas éticos. Neste sentido, a implantação da fila única para o atendimento aos pacientes de COVID-19, em discussão na Câmara dos Deputados, se aprovada, parece poder contribuir para aliviar o fardo que cai sobre os profissionais. O Projeto de Lei № 2.176/2020 que cria uma fila única em unidades públicas ou privadas de terapia intensiva (UTI) leva em conta apenas a gravidade do caso e a ordem de chegada. E hoje, de acordo com o Cadastro Nacional de Estabe- lecimentos de Saúde (CNES), o Sistema Único de Saúde (SUS) conta com $44 \%$ destes leitos no país, sendo os outros $56 \%$ privados $^{10-11}$.

Os trabalhadores da Enfermagem assistiram ao achatamento salarial nas últimas décadas, em uma proporção que os colegas médicos, por exemplo, não experimentaram. Ainda que representem $55 \%$ dos profissionais do setor público, perdem o poder de negociação, sobretudo por a Enfermagem não ter representatividade política nem gerencial em nível Municipal, Estadual e Federal. Os gestores que estão no poder alegam que aumentos expressivos não cabem nos cofres, mas não explicam nem justificam suas decisões e, assim a essencialidade continua em segundo plano.

A pandemia, contudo, expôs de forma incontestável o quanto os trabalhadores da Enfermagem são essenciais. Todas as ações de contingenciamento na pandemia dependeram das ações de cuidado e gestão feitas por enfermeiros, técnicos e auxiliares de enfermagem, em todos os serviços de saúde do país. É urgente a aprovação do pagamento de insalubridade para a categoria e a pensão para familiares dos que foram a óbito em serviço.

O Congresso Nacional neste contexto deve discutir o Piso Salarial Nacional e tirar da gaveta o Projeto de Lei № 2.295/2000, que trata da "Jornada de 30 horas"12, proposta e aprovada em todas as comissões, mas que alcançou a maioridade sem votação, refletindo a invisibilidade política das pautas relacionadas à profissão.

A valorização do profissional de Enfermagem passa também, necessariamente, por uma repactuação do financiamento do SUS, que vem de subfinanciamento histórico, que foi severamente agravado pela Emenda Constitucional № 95, que limita, por duas décadas, o investimento público.

Quanto ao estímulo profissional, o COFEN vem ofertando capacitação, com cursos à distância para 300 mil profissionais de Enfermagem, em parceria com a Universidade Federal de Santa Catarina (UFSC), e vem atuando fortemente na pandemia, publicando informações técnicas e orientações atualizadas, de acordo com o avanço de conhecimento da doença ${ }^{13}$.

$\mathrm{Na}$ prática, o reconhecimento e o respeito é o único anseio dos profissionais amplamente atendidos nesse momento. Técnicos, auxiliares e enfermeiros são aplaudidos em todas as partes do mundo. A solidariedade aos profissionais da saúde, porém, não deve se manifestar apenas por meio de aplausos, mas também ao diminuir a sua carga de estresse, com medidas que favoreçam o apoio emocional.

O que é essencial para os profissionais de Enfermagem, mais do que nunca, deve ser tratado como essencial pela sociedade e pelos gestores em todas as esferas de gover- 
no. É hora de colocar a Enfermagem na linha de frente das prioridades do sistema de saúde brasileiro.

\section{Limitações do Estudo}

Apenas um processo reflexivo sobre os dados encontrados pelo COFEN foi desenvolvido neste estudo. Outras análises merecem ser desenvolvidas para ampliar a compreensão desta temática.

\section{Contribuições para A Prática}

Estudar condições de trabalho tem sido prática de diferentes pesquisadores, todavia conhecer de forma anônima o que preocupa os profissionais de Enfermagem certamente auxilia a direcionar ações e políticas públicas.

\section{CONCLUSÃO}

A despeito da visibilidade conquistada por sua atuação na luta contra o coronavírus, os profissionais de Enferma- gem necessitam de maior reconhecimento do poder público. Questões há muito reivindicadas como salário adequado, insalubridade, jornada de trabalho de 30 horas, bem como melhores condições de trabalho, estão entre as propostas para garantir o que é essencial para os trabalhadores essenciais, durante e depois desta pandemia.

A qualidade da assistência à saúde não pode ser dissociada das condições de vida e trabalho dos profissionais de Enfermagem, que representam mais da metade dos trabalhadores da Saúde no Brasil. A pandemia de COVID-19 convida a sociedade brasileira a repactuar prioridades, reafirmando a importância da assistência universal à saúde, conquista civilizatória do século $X X$, que chegou tardiamente ao país e foi posta em xeque nos últimos anos, e dos trabalhadores essenciais para a efetivação deste direito.

Contribuições dos Autores: ICKOC e NPF desenvolveram juntos todas as etapas do estudo.

\section{REFERÊNCIAS}

1. World Health Organization (WHO). WHO DirectorGeneral's statement on IHR Emergency Committee on Novel Coronavirus (2019-nCoV). [Internet]. 2020 Jan 30 [cited 2020 June 12]; Available from: https://www.who.int/ dg/speeches/detail/who-director-general-s-statement-onihr-emergency-committee-on-novel-coronavirus-(2019ncov)

2. World health Organization (WHO). WHO DirectorGeneral's opening remarks at the media briefing on COVID-19. [Internet] 2020 Mar 11 [cited in 2020 June 12]; Available from: https://www.who.int/dg/speeches/detail/ who-director-general-s-opening-remarks-at-the-mediabriefing-on-covid-19---11-march-2020.

3. Conselho Federal de Enfermagem (COFEN). Enfermagem em Números. [Internet]. 2020 [cited in 2020 Jul 3]. Available from: http://www.cofen.gov.br/enfermagem-em-numeros.

4. Conselho Federal de Enfermagem (COFEN). Observatório da Enfermagem [Internet]. 2020 [cited in 2020 Jul 3]. Available from: http://observatoriodaenfermagem.cofen. gov.br/.

5. Conselho Federal de Enfermagem (COFEN). [Internet] 2020 [cited in 2020 Jun 18]. Available from: http://ouvidoria. cofen.gov.br/cofen/transparencia/38805/download/PDF.

6. Machado M, Oliveira E, Lemos W, Lacerda W, Filho W, Wermelinger $M$ et al. Mercado de trabalho da Enfermagem: aspectos gerais. Enferm Foco [Internet] 2016 [cited in 2020 Jun 18]; 7(esp.):35-53. Available from: http://revista.cofen. gov.br/index.php/enfermagem/article/view/691/301.

7. The Lanced. Souadka A, Essangri H, Benkabbou A, Amrani
L, Majbar MA. COVID-19 and Healthcare worker's families: behind the scenes of frontline response, EClinicalMedicine [Internet] 2020 May 3 [cited in 2000 Jun 18]; 23 (2020):100373 Available from: https://www.ncbi.nlm.nih gov/pmc/articles/PMC7196421/.

8. Organização Pan-Americana da Saúde (OPAS). Checklist para a gestão dos recursos Humanos em saúde em resposta à COVID-19. [Internet]. 2020 [cited in 2000 Jun 18]. Available from: https://iris.paho.org/bitstream/ handle/10665.2/52158/OPASBRAHSSHRCOVID19200011_ por.pdf?sequence=18isAllowed=y.

9. Organização Internacional do Trabalho (OIT). COVID-19 and the world of work. Fourth edition Updated estimates and analysis. [online] 2020 [viewed 30 May 2020]. Available from: https://www.ilo.org/wcmsp5/groups/ public/---dgreports/---dcomm/documents/briefingnote/ wcms_745963.pdf

10. Câmara dos Deputados (BR). Projeto de Lei № 2.176/2020. [Internet] 2020 [cited in 2000 Jun 18]. Available from: https://www.camara.leg.br/propostaslegislativas/2250593.

11. Projeto de lei cria fila única em UTIs para pacientes com Covid. Portal Medicina S/A [online]. 2020 [viewed 15 May 2020]. Available from: https://medicinasa.com.br/ fila-unica-covid/

12. Brasil. Câmara dos Deputados [online]. Projeto de Lei 2295/2000. 2020 [viewed 17 May 2020]. Available from: https://www.camara.leg.br/propostas-legislativas/17915

13. Atualiza Enfermagem [online]. UFSC-Cofen. 2020 [viewed 18 May 2020]. Available from: https:// atualizaenfermagemcovid19.ufsc.br/ 